\title{
High-amylose Rice: Starch Molecular Structural Features Controlling Cooked Rice Texture and Preference ${ }^{+}$
}

\author{
Keyu Tao ${ }^{1}$, Wenwen Yu ${ }^{2}$, Prakash Sangeeta ${ }^{3}$, Robert Goulston Gilbert 1,* \\ 1 Queensland Alliance For Agriculture and Food Innovation (qaafi), The University of Queensland, \\ St Lucia, QLD 4067, Australia; k.tao@uq.net.au \\ 2 Department of Food Science \& Engineering, Jinan University, Guangzhou 510632, China; \\ wen.yu2@uq.net.au \\ 3 School of Agriculture and Food Science, The University of Queensland, St Lucia, QLD 4067, Australia; \\ s.prakash@uq.edu.au \\ * Correspondence: b.gilbert@uq.edu.au \\ + Presented at the third International Tropical Agriculture Conference (TROPAG 2019), Brisbane, Australia, \\ 11-13 November 2019.
}

Published: 21 January 2020

\begin{abstract}
Cooked high-amylose rices have slower digestibility, giving nutritional benefits, but inferior eating qualities. In this study, Rapid Viscosity Analysis, quantitive descriptive sensory analysis with all panellists from China and Textural Profile Analyser (TPA) have been used to measure rice texture and eating quality of cooked rice. Molecular structural mechanisms for this inferior eating quality are found here using structural analysis by size-exclusion chromatography of both the parent starch and starch leached during cooking. All commonly-accepted sensory attributes of cooked rice were characterized by a trained human panel. Hardness, with the strongest negative correlation with panelist preference, is the dominant but not sole factor determining palatability. Rice with larger amylopectin size can bond more water, thereby have lower hardness value. Meanwhile, hardness is controlled by the amounts of medium and long amylopectin chains and amylose in the starch, and by amylose content and amount of longer amylopectin chains in the leachate. With this, it is concluded for the first time that rice containing 19 25\% amylose content are most preferred by the panel. Meantime, it is showed that breakdown viscosity and swelling power of native rice flour can be and should be used as indicators for predicting rice eating quality. This gives knowledge and understanding of the molecular structural characteristics of starch controlling cooked-rice preference: not just high amylose but also other aspects of molecular structure. This can help rice breeders to target starch-synthesis genes to select slowly digested (healthier) rices with acceptable palatability.
\end{abstract}

Keywords: rice; starch; eating quality; size-exclusion chromatography; molecular structure; preference

Funding: This research was funded by a National Natural Science Foundation of China, grant number C1304013151101138 and the 2017 Jiangsu Innovation and Entrepreneurship talents program (to R.G.G.).

Acknowledgments: We appreciate useful advice from Jixun Luo (CSIRO Plant Industry) and Hongyan Li (Beijing Technology \& Business University), and the New South Wales Department of Primary Industries for providing rice samples. 
Conflicts of Interest: The author declares no conflict of interest.

(C) 2020 by the authors. Licensee MDPI, Basel, Switzerland. This article is an open access article distributed under the terms and conditions of the Creative Commons Attribution (CC BY) license (http://creativecommons.org/licenses/by/4.0/). 